Article

\title{
In situ Investigation of Titanium Powder Microwave Sintering by Synchrotron Radiation Computed Tomography
}

\author{
Yu Xiao ${ }^{1}$, Feng $\mathrm{Xu}^{1, *}, \mathrm{Xiaofang} \mathrm{Hu}^{1}$, Yongcun $\mathrm{Li}^{2}{ }^{2}$, Wenchao Liu ${ }^{1}$ and Bo Dong ${ }^{1}$ \\ Received: 3 October 2015; Accepted: 23 December 2015; Published: 4 January 2016 \\ Academic Editor: Klaus-Dieter Liss \\ 1 CAS Key Laboratory of Mechanical Behavior and Design of Materials, Department of Modern Mechanics, \\ University of Science and Technology of China, Hefei 230026, China; xiaoyuxy@mail.ustc.edu.cn (Y.X.); \\ huxf@ustc.edu.cn (X.H.); liuwc@mail.ustc.edu.cn (W.L.); dongbo@mail.ustc.edu.cn (B.D.) \\ 2 Department of Mechanics, Taiyuan University of Technology, Taiyuan 030024, China; liyongcun@tyut.edu.cn \\ * Correspondence: xufeng3@ustc.edu.cn; Tel.: +86-551-6360-0564; Fax: +86-551-6360-6459
}

\begin{abstract}
In this study, synchrotron radiation computed tomography was applied to investigate the mechanisms of titanium powder microwave sintering in situ. On the basis of reconstructed images, we observed that the sintering described in this study differs from conventional sintering in terms of particle smoothing, rounding, and short-term growth. Contacted particles were also isolated. The kinetic curves of sintering neck growth and particle surface area were obtained and compared with those of other microwave-sintered metals to examine the interaction mechanisms between mass and microwave fields. Results show that sintering neck growth accelerated from the intermediate period; however, this finding is inconsistent with that of aluminum powder microwave sintering described in previous work. The free surface areas of the particles were also quantitatively analyzed. In addition to the eddy current loss in metal particles, other heating mechanisms, including dielectric loss, interfacial polarization effect, and local plasma-activated sintering, contributed to sintering neck growth. Thermal and non-thermal effects possibly accelerated the sintering neck growth of titanium. This study provides a useful reference of further research on interaction mechanisms between mass and microwave fields during microwave sintering.
\end{abstract}

Keywords: microwave sintering; microstructure; synchrotron radiation computed tomography

\section{Introduction}

As an advanced material preparation method, microwave sintering has been extensively investigated because of its numerous advantages, such as high heating rate, overall and even heating, and material microstructure improvement. This technique is initially applied to nonmetallic material sintering. Microwaves were initially believed to be unable to heat metal materials because of the reflection at surfaces. Since its first application performed by Roy et al. in 1999, microwave sintering of metal, alloy, and metallic glassy powder has been investigated [1-4]. This technique can promote particle size uniformity and densification rate; it can also improve the macro-properties of materials to a greater extent than conventional sintering. For example, Roy et al. [1] confirmed that the modulus of Fe-Ni rupture is $60 \%$ higher than that of conventional specimens after 10-30 min of microwave treatment. These excellent advantages are attributed to the combination of thermal and non-thermal effects. However, the definite mechanisms of microwave sintering remain unclear. Janney et al. [2] indicated that the activation energy is lower in microwave sintering than in conventional sintering. Conversely, Saitou $\mathrm{K}$ et al. [3] demonstrated that the microwave field does not affect activation energy during sintering. Other theories, including eddy currents [5], crystallization enhancement [6], 
and micro-focusing and polarization effects [7], have been proposed. However, direct experimental evidence is difficult to obtain; as such, these theories cannot be easily verified because of the extreme experimental conditions required during microwave sintering.

The macro-properties of materials are determined on the basis of their microstructures which are driven by mechanisms of the sintering kinetics mechanisms. Relevant microwave sintering mechanisms can be revealed by continuous in situ tracking of the internal microstructure evolution. After determining all the microstructure characteristics, microwave heating mechanisms and sintering kinetics can be further analyzed. However, studies adopting conventional testing techniques have yet to observe microwave sintering in situ because of the experimental limitations of conventional testing techniques, such as optical microscopy, transmission electron microscopy, and scanning electron microscopy. Therefore, in situ and non-destructive testing techniques [8], such as synchrotron radiation computed tomography (SR-CT) [9], should be developed.

SR-CT is a novel testing technology that can achieve non-destructive and real-time 3D observations in extreme environments, such as high or low temperature, high pressure, and intense radiation. For example, Bale et al. [10] conducted a real-time study of microstructure behavior under mechanical loading at $>1600{ }^{\circ} \mathrm{C}$. When applied in sintering studies, SR-CT can continuously obtain accurate experimental data of surface and internal 3D microstructure evolution during sintering without interrupting the process and destroying the sample. Quantitative analysis can then be conducted on the basis of SR-CT experimental data. With these excellent advantages, SR-CT is quite suitable for the in situ investigation of sintering.

In this study, SR-CT was applied to investigate titanium powder microwave sintering in situ. $2 \mathrm{D}$ and $3 \mathrm{D}$ images at different sintering times were reconstructed. In the images of internal microstructure evolution, sintering phenomena included sintering neck growth, powder densification, and pore closing, which commonly occur in conventional sintering. Unique phenomena, such as particle smoothing, rounding, and short-term growth, contacted particles being isolated, which rarely occur in conventional sintering, were also observed. These phenomena may be attributed to eddy current loss, micro-focusing, and interfacial polarization effect. Our experimental data revealed the sample microstructure characteristics, such as surface area and sintering neck size. The kinetic mechanisms of sintering neck growth were quantitatively analyzed and compared with those of other metal microwave sintering techniques. Our results revealed that sintering kinetic behaviors were different from those of aluminum in early and intermediate periods. The cause of this difference was investigated, and the corresponding sintering mechanisms were analyzed. Results showed that the sintering behaviors were quite different during microwave sintering because of the heterogeneity between titanium and aluminum. Eddy current loss, other heating mechanisms, and non-thermal effects in microwave sintering were also observed.

\section{Experimental Section}

The microwave sintering experiment was conducted in a BL13W1 beam line at Shanghai Synchrotron Radiation Facility (Shanghai, China); the beam line is a third-generation light source with excellent features, including high intensity, high brilliance, high polarization rate, and quasi-coherence. The charge-coupled device resolution was $0.74 \mu \mathrm{m}$ per pixel. Chemically pure titanium powder with an average particle size of approximately $23 \mu \mathrm{m}$ and 99.9\% purity (Aladdin Biochemical Technology Co., Ltd., Shanghai, China) was used. Acid pickling was conducted to remove oxides on the particle surfaces. The powder was dried in a vacuum oven at $120^{\circ} \mathrm{C}$ and then loosely poured into a capillary tube with an inner diameter of approximately $0.3 \mathrm{~mm}$ and a height of approximately $10 \mathrm{~mm}$. Some copper particles were fixed on the outer capillary surface as marking points to easily locate the same region at different sintering times. The sample was then introduced to a specially designed microwave sintering furnace with a multimode cavity of $2.45 \mathrm{GHz}$ and an output power of $0-3 \mathrm{~kW}$. An SiC susceptor was used to preserve heat and to accelerate the increase in sample temperature because of the small sample size and large space of the multimode cavity chamber. The top of the sample at the 
far end of the susceptor was chosen as the test region for the SR-CT experiment to reduce the influence of the susceptor (Figure 1). The temperature was measured using a thermo tracer (type TH5104; NEC Corp., Tokyo, Japan) under the following parameters: temperature measurement range from $-10^{\circ} \mathrm{C}$ to $1500{ }^{\circ} \mathrm{C}$, accuracy of $\pm 1.0 \%$ (full scale), and emissivity of 0.6 . The temperature increased to $900{ }^{\circ} \mathrm{C}$ within approximately $20 \mathrm{~min}$ and remained constant for $60 \mathrm{~min}$. Argon was used as a protective atmosphere. The experimental facility is shown in Figure 1. We compared the proposed technique with a previously described aluminum microwave sintering [11] that requires the same parameters as the microwave sintering furnace.

The furnace chamber must be an open space to allow the rotation system (Figure 1) to dive into the furnace because sample rotation is necessary to obtain computed tomography data. Therefore, the protective atmosphere must be constantly pumped into the furnace. The gas flow rate was set at a very slow limit to avoid excessive heat loss from the hole of the rotation system.

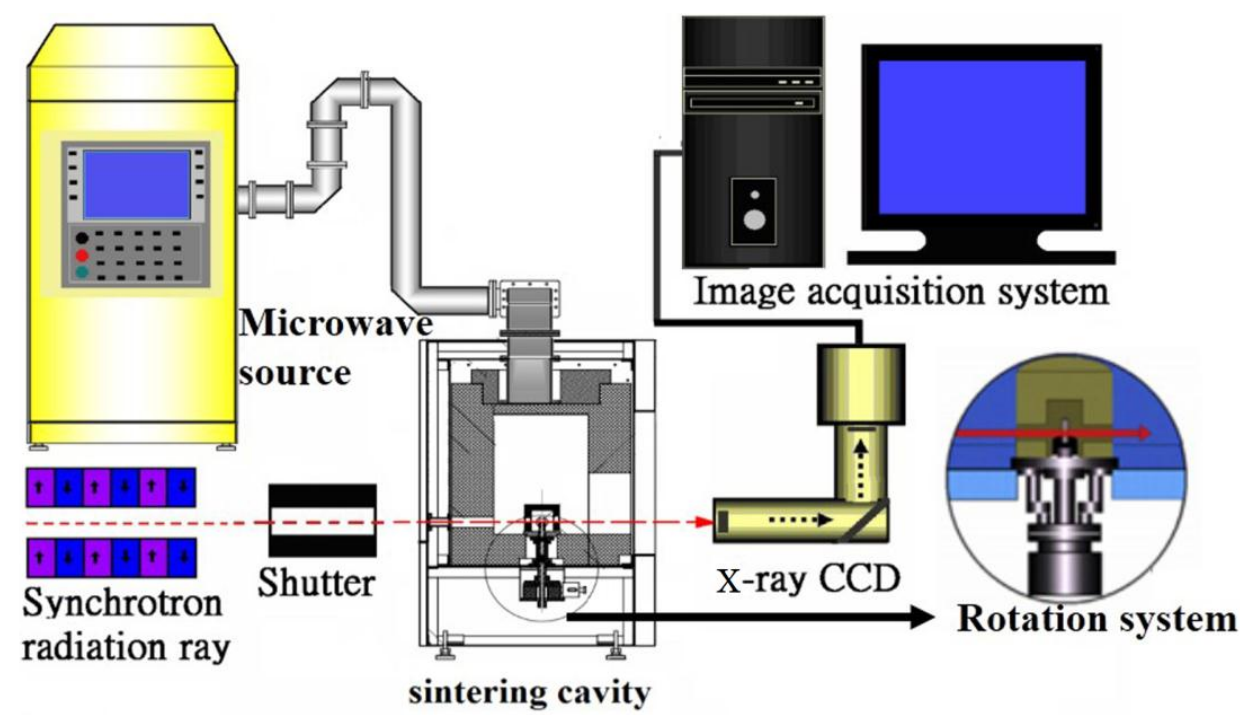

Figure 1. Schematic of in situ synchrotron radiation computed tomography (SR-CT) system of microwave sintering.

\section{Results}

Figure 2 shows the 3D images of microstructure evolution at different times during microwave sintering. Sintering phenomena, such as particle densification and sintering neck formation and growth, were observed. Further analysis was conducted on the basis of 2D images to present internal microstructure evolution and to obtain morphological parameters.

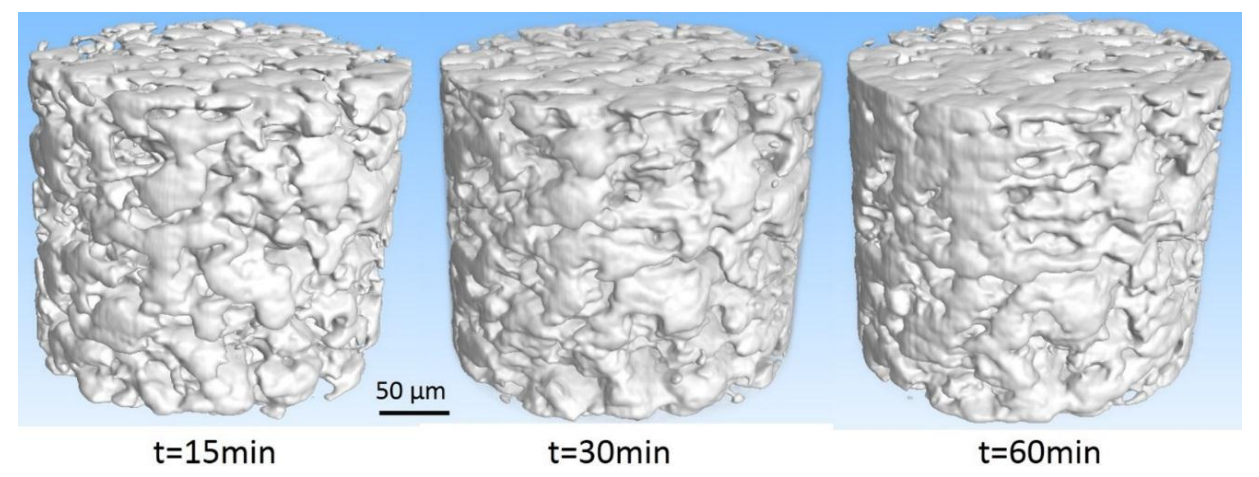

Figure 2. 3D images of the microstructure at different sintering times. 
The images shown in Figure 3 were reconstructed by using the filtered back projection algorithm. The cross-section images of the same internal areas at different sintering times were obtained on the basis of marking particles present on the sample and related algorithms. Grayscale ranges from 0-255. As the grayscale value approaches 255, X-ray is increasingly absorbed; the relative density is also high. This trend indicates that white regions represent particles and black regions represent pores. These cross-section images also reveal typical sintering phenomena similar to conventional sintering: (1) the particles in the circle contacted with each other while sintering neck formed and grew and (2) the pores marked with red asterisks closed and their size decreased.

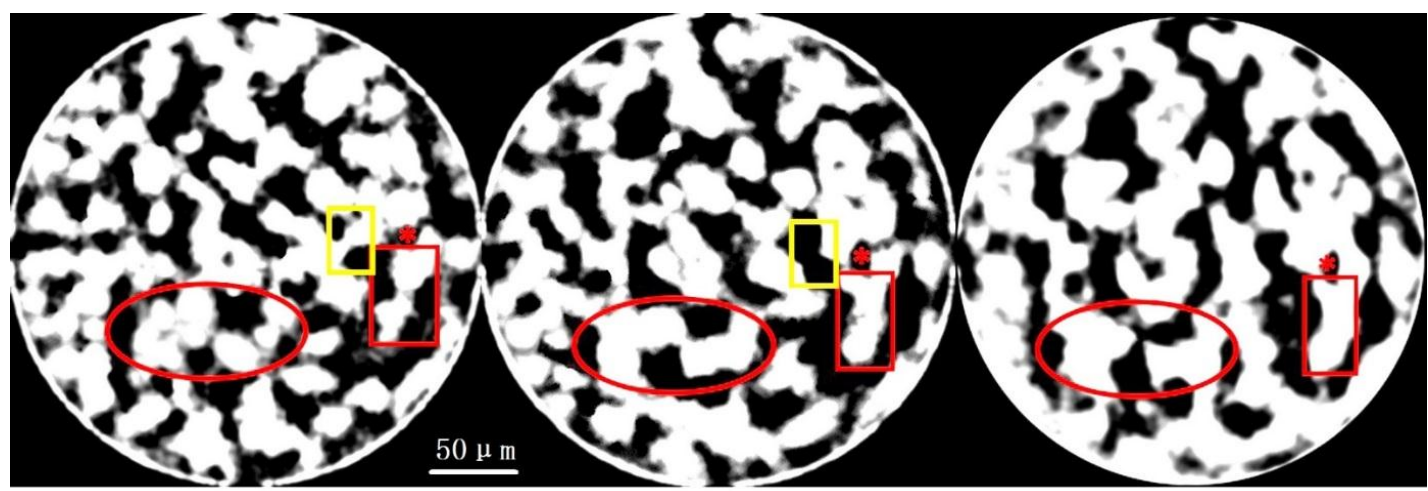

(a) $\mathrm{t}=15 \mathrm{~min}$

(b) $t=30 \mathrm{~min}$

(c) $\mathrm{t}=60 \mathrm{~min}$

Figure 3. Microstructure of the same cross section at different sintering times of $15 \mathrm{~min}(\mathbf{a}), 30 \mathrm{~min}(\mathbf{b})$ and $60 \mathrm{~min}(\mathbf{c})$.

Special microwave sintering phenomena which were similar and different with other kinds of metals and rarely seen in conventional sintering were captured and shown in Figure 3.

(1) The particles in the red rectangle grow more spherically and smoothly within a short time as the corners and burrs on the surface disappear. A similar process can also be observed in the circle. This phenomenon may be attributed to the loss of eddy current on the particle surface; as a result, mass diffusion is accelerated. Furthermore, non-thermal effects, such as interfacial polarization between grain surface and pores, and micro-focusing effects at the corner and burrs likely contribute to particle smoothing and rounding processes. Therefore, the proposed process was much faster than conventional sintering.

(2) The two large particles in the red circle shown in Figure $3 b$ come in contact with each other when the sintering neck is formed. However, the two particles are isolated from each other instead of sintering together (Figure 3c) as a consequence of the tensile and pressure from other particles. The particles in the yellow rectangle also exhibit similar behaviors. This phenomenon may have been caused by the micro-focusing effect. During microwave distribution, local microwave fields are disproportionately strong in certain regions because of the focusing influences of the microstructure, such as sintering neck, particle boundaries, and rough surfaces. Thus, non-uniform energy deposition occurs and temperature remarkably increases. The connecting region of two particles may be melted by the local high temperature attributed to the micro-focusing effect and then be broken by the tensile and pressure from other particles.

(3) Several small particles in the red circle shown in Figure 3a quickly grow together into two large particles within $15 \mathrm{~min}$. This phenomenon may have been caused by the acceleration of mass diffusion as a result of the micro-focusing effect at the sintering neck regions.

These unique microwave sintering phenomena can be rarely observed in conventional sintering but can be detected in other microwave sintering experiments of metal and ceramic-metal mixtures; these phenomena are attributed to non-thermal effects. Phenomenon (1) also occurs in the microwave sintering of aluminum [11]. This phenomenon indicates that some common mechanisms occur 
during microwave sintering of different kinds of metals, such as titanium and aluminum. However, phenomena (2) and (3) were not observed in the microwave sintering of aluminum; therefore, different mechanisms are associated with the microwave sintering of different metals. These two phenomena were captured in the microwave sintering of the $\mathrm{Al}-\mathrm{SiC}$ mixed system, which showed different microwave sintering mechanisms from those of pure aluminum [12]. The morphological parameters were quantitatively analyzed to further investigate the different microwave sintering mechanisms of titanium compared with other metal of aluminum.

The particles on the same cross-section at the initial height may migrate to the cross-section at other heights during the sintering experiment. Not all of the microstructure features can be traced in Figure $3 a-c$ because of the thermal expansion and traction among the particles. However, the particles can be observed in the $3 \mathrm{D}$ images.

\section{Discussion}

Interaction mechanisms, such as thermal effects and non-thermal effects between mass and microwave fields, remarkably influence microstructure evolution. Morphological parameters, such as sintering neck growth rate and particle surface area, were obtained and quantitatively analyzed to examine kinetic mechanisms.

Sintering neck formation and growth were quantitatively evaluated. In traditional sintering theories, the dynamics of stable neck growth summarized by Kuczynski [13] is shown as follows. This theory was applied to quantify the sintering neck growth rate during microwave sintering and to provide a reference on the corresponding mass diffusion mechanisms.

$$
\left(\frac{x}{a}\right)^{n}=\frac{F(t)}{a^{m}} t
$$

where $x / a$ is the ratio of inter-particle neck radius to the particle radius; $F(t) / a^{m}$ is a constant that involves particle size, temperature, and geometric and material terms; $t$ is the sintering time; and $n$ is the sintering neck growth exponent. Equation (1) indicates that the plot of $\log (x / a)-\log (t)$ shows a linear relationship with a slope equal to $1 / n$. According to the exponential criterion, different $n$ represents different major diffusion mechanisms. Watershed algorithm was used to reveal the sintering-neck kinetic mechanisms and to obtain the morphological parameters. Sintering neck extraction is illustrated in Figure 4. The sintering neck between two particles can be distinguished and the size of sintering neck can be counted [14]. A total of 100 cross-sections of the same region at different sintering times identified by the marking points and microstructure features were selected and subjected to statistical analyses. The same watershed operation was applied to these cross-sections, and the size of the sintering neck was the average value of the selected region.

The line $\log (x / a)-\log (t)$ is shown in Figure 4. For comparisons, the line of aluminum microwave sintering in the present work [11] is also shown in Figure 5b.

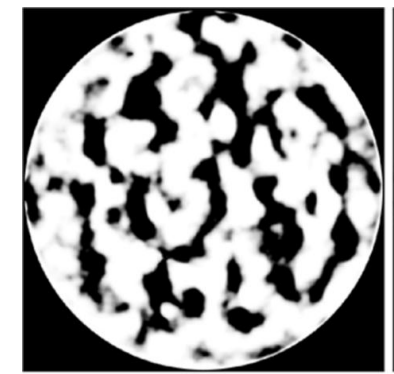

(a)

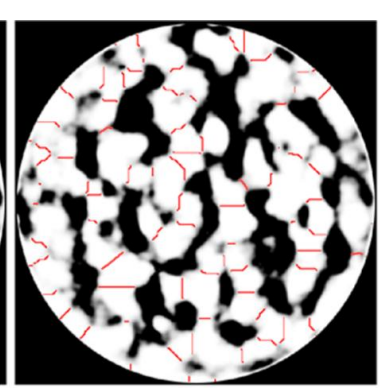

(b)

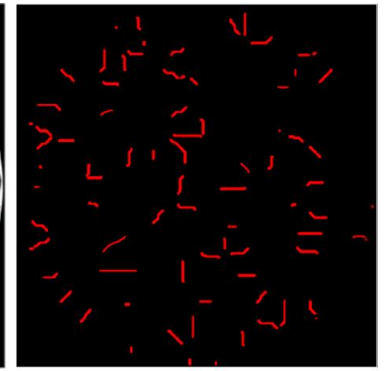

(c)

Figure 4. Sintering neck extraction by watershed method: The cross section image (a), the result of watershed algorithm (b), and the extracted sintering necks (c). 


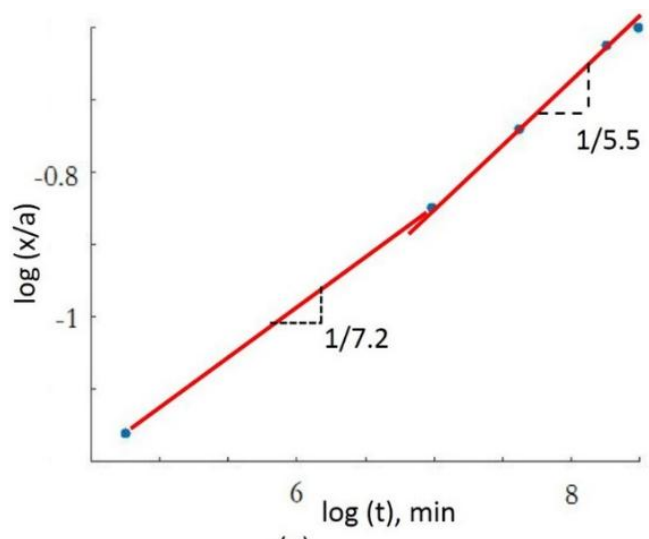

(a)

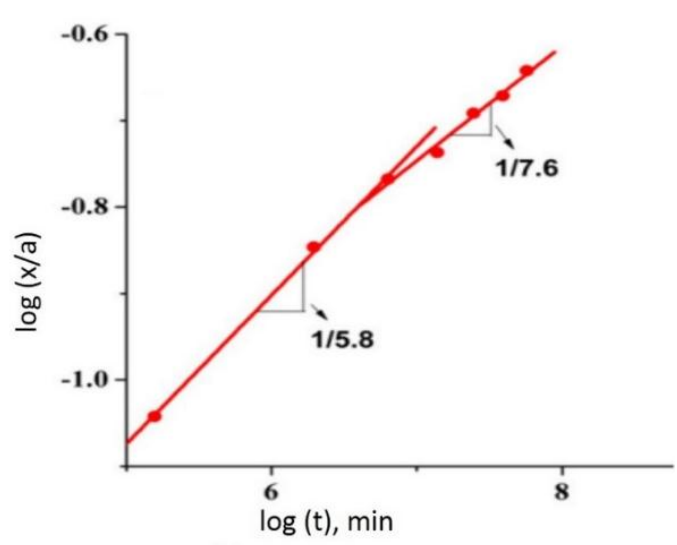

(b)

Figure 5. Sintering neck growth curves at $\log (x / a)-\log (t)$ of $\mathrm{Ti}(\mathbf{a})$ and $\mathrm{Al}(\mathbf{b})$.

Figure 5 shows that the scatter diagram of $\log (x / a)-\log (t)$ for both titanium and aluminum satisfies the linear relationship, but the differences are also significant. In the early period of microwave sintering, $n$ indicated that the sintering neck growth rate of titanium was slower than that of aluminum, and the dominant diffusion mechanisms of titanium and aluminum were surface diffusion and volume diffusion, respectively. As sintering proceeded, the sintering neck growth became quite different from the intermediate period. The sintering neck growth rate of titanium significantly accelerated in the intermediate period; by contrast, the sintering neck growth rate of aluminum decelerated. The dominant diffusion mechanism was also different, and $n$ indicated that volume diffusion and surface diffusion were the dominant mechanisms of titanium and aluminum, respectively. These results revealed that the sintering processes of the two metals behaved differently. The reasons for these phenomena were analyzed on the basis of the interaction mechanisms between mass and microwave fields.

We discussed the difference in the sintering neck growth rate in the early period. The major heating mechanism of metal powder in the microwave field is eddy current loss on the particle surface [15]. The power loss of metal particle is in accordance with the following equation [16,17]:

$$
P=\frac{1}{2} \int \vec{E} \cdot \vec{J}_{\mathrm{s}} \mathrm{d} V=\frac{1}{2} \sigma \int\left|\vec{J}_{\mathrm{s}}\right|^{2} \mathrm{~d} V
$$

where $J_{\mathrm{s}}$ is the surface current calculated as $\overrightarrow{J_{\mathrm{s}}}=\vec{n} \times \vec{H}_{\mathrm{t}} ; \vec{H}_{\mathrm{t}}$ is the tangential magnetic vector; and $\sigma$ is the conductance. The eddy current loss of aluminum was much higher than that of titanium because the electrical conductivity of aluminum (approximately $35.5 \times 10^{6} \mathrm{~S} / \mathrm{m}$ ) is higher than that of titanium (approximately $2.6 \times 10^{6} \mathrm{~S} / \mathrm{m}$ ). Therefore, aluminum yields a faster sintering neck growth rate than titanium in the early period, as shown in the sintering neck growth curve.

The sintering neck growth of titanium accelerated, but the sintering neck growth of aluminum decelerated when $\log (x / a)$ reached approximately -0.8 . In the previous work, alumina possibly covers the grain surface and hinders mass diffusion to slow down sintering neck growth. A further analysis was conducted to clarify the acceleration of the sintering neck growth rate of titanium from the intermediate period.

The eddy current was located on a very thin layer of the particle surface because of the restriction of the skin depth of microwave (approximately $7 \mu \mathrm{m}$ for titanium at $2.45 \mathrm{GHz}$, less than the size of one particle). Therefore, the total free surface area of particles significantly influences the eddy current loss [12]. Figure 6 reveals the statistical results of the total particle free surface area as the average values of the same several layers. Figure 6 also shows that the surface area reduced rapidly in the early period. However, the surface area remarkably decreased as the sintering process progressed. 
Figure 5 a indicates that the eddy current loss was reduced in the intermediate period of the sintering process; by contrast, the sintering neck growth rate accelerated. In addition to eddy current loss, other heating mechanisms caused by thermal and non-thermal effects occurred, and these mechanisms also contributed to the acceleration of sintering neck growth. Other possible reasons for the sintering neck growth acceleration were proposed.

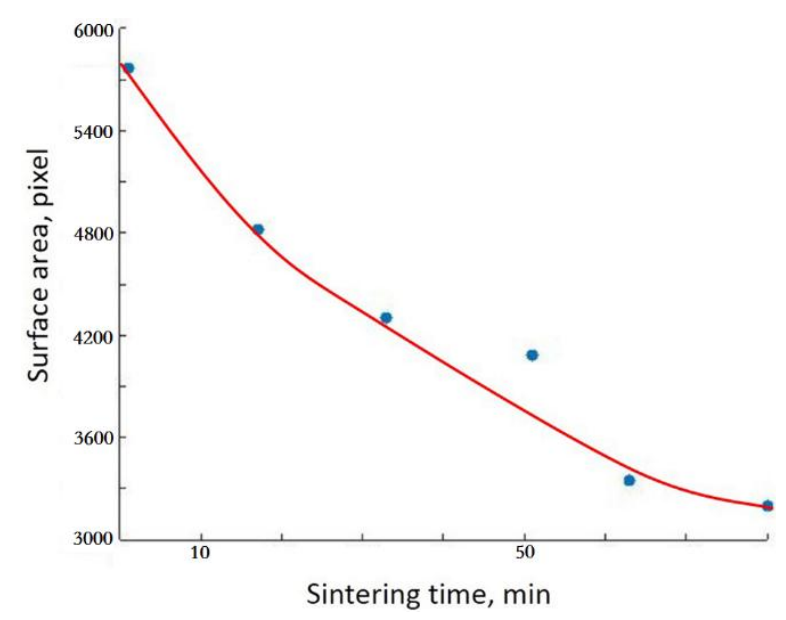

Figure 6. Variation in the total particle free surface area.
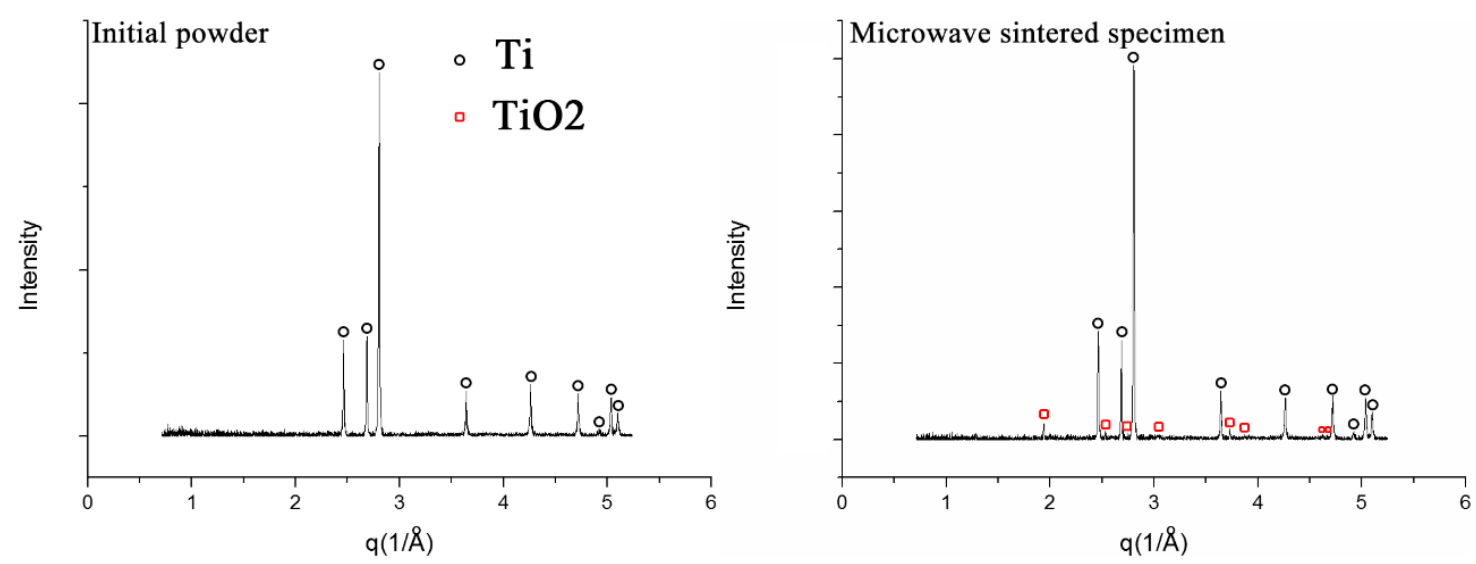

Figure 7. X-ray diffraction patterns of the powder and specimen.

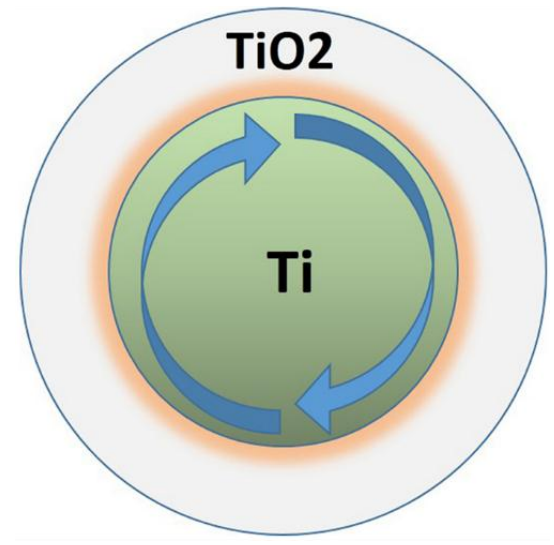

Figure 8. Schematic of particle loss. 
(1) Oxidation may occur to a certain extent because of the constraints in experimental conditions, such as open furnace chamber and slow protective atmosphere flow rate, although sintering proceeded in an argon-protected atmosphere. Titanium dioxide formed and covered the grains as sintering proceeded because of the slow protective gas flow rate. The X-ray diffraction patterns of the powder before and after microwave sintering are represented in Figure 7. Oxide was not detected in the initial powder before sintering. By contrast, the diffraction peaks of titanium dioxide appeared, and this finding indicated the formation of oxide after microwave sintering was completed. The dominant microwave heating mechanism of the pure metal powder was eddy current loss. When titanium dioxide covered the metal particles (Figure 8 showing the thickness of titanium dioxide as a representation, not the actual thickness), the inner metal was heated by the eddy current and the outer titanium dioxide was heated by dipolar polarization loss. Therefore, the total power loss of metal oxides may be higher than that of pure metal particles; as a result, the sintering neck of titanium grew rapidly from the intermediate period. In addition, the sintering neck growth of aluminum decelerated in the present work because of the poor microwave-absorbing property of alumina.

(2) When titanium dioxide was formed, polarization charges accumulated at the interface of metal and oxide because of the heterogeneity between titanium and titanium dioxide. Therefore, the interfacial polarization effect cannot be ignored as another important heating mechanism. The heating effect caused by interfacial polarization probably accelerated the sintering neck growth. For the microwave sintering of aluminum, the difference between the interface of aluminum-alumina and titanium-titanium dioxide resulted in different strengths of interfacial polarization. Therefore, the sintering neck growth of aluminum decelerated in the present work.

(3) Local plasma-activated sintering [18] promoted the sintering neck growth. The local electromagnetic field may be strong in some regions of the sintering neck, pores, and burrs of the rough surface because of the focusing influence of the microstructure; thus, the protective atmosphere of argon was ionized at the local regions. Evaporation-condensation mechanisms may be the local dominant diffusion mechanism because of plasma-generated ultra-high temperature; this mechanism likely accelerates the sintering neck growth and densification. Moreover, the atomic radius of most air components is smaller than that of argon; as such, the ionization energy of air is higher than that of argon and the ionization of air becomes more difficult than that of argon. Therefore, the microwave sintering of aluminum was not accelerated.

\section{Conclusions}

SR-CT was applied to investigate titanium powder microwave sintering in situ. The cross-sections of the same microstructure features at different sintering times were extracted on the basis of the reconstructed images. The sintering phenomena differ from conventional sintering, such as particle smoothing and rounding and contacted particles isolation. These unique phenomena may be attributed to the thermal and non-thermal effects of microwave field, including eddy current, interfacial polarization effect, and micro-focusing effect. The microstructure morphological parameters, including sintering neck size and particle surface area, were obtained and quantitatively analyzed to examine the different sintering mechanisms of the two kinds of aluminum and titanium metals. Our results revealed that the sintering neck growth rate accelerated from the intermediate period. Considering that the particle-free surface area associated with the eddy current loss was reduced, we can infer that other heating mechanisms, in addition to eddy current loss, occurred. Possible reasons, such as mixing heating of eddy current loss, dielectric loss, interfacial polarization, and local plasma-activated sintering, were proposed. The microwave sintering of titanium was enhanced because of these interaction mechanisms, including thermal and non-thermal effects.

Acknowledgments: This research was supported by the National Natural Science Foundation of China (No. 11272305, No. 11172290, No. 11472265, No. 11402160, No. 10902108) and the National Basic Research Program of China (973 Program, No. 2012CB937504) and Anhui Provincial Natural Science Foundation (No. 1508085MA17). 
Author Contributions: Yu Xiao, Feng Xu and Yongcun Li analyzed the experimental data and wrote the paper; Feng $\mathrm{Xu}$ and Xiaofang Hu conceived and designed the experiment; Yu Xiao, Wenchao Liu and Bo Dong prepared the sample and performed the experiment.

Conflicts of Interest: The authors declare no conflict of interest.

\section{References}

1. Roy, R.; Agrawal, D.; Cheng, J.; Gedevanishvili, S. Full sintering of powdered-metal bodies in a microwave field. Nature 1999, 399, 668-670.

2. Janney, M.A.; Kimrey, H.D.; Allen, W.R.; Kiggans, J.O. Enhanced diffusion in sapphire during microwave heating. J. Mater. Sci. 1997, 32, 1347-1355. [CrossRef]

3. Saitou, K. Microwave sintering of iron, cobalt, nickel, copper and stainless steel powders. Scr. Mater. 2006, 54, 875-879. [CrossRef]

4. Chhillar, P.; Agrawal, D.; Adair, J.H. Sintering of molybdenum metal powder using microwave energy. Powder Metall. 2008, 51, 182-187. [CrossRef]

5. Ma, J.; Diehl, J.F.; Johnson, E.J.; Martin, K.R.; Miskovsky, N.M.; Smith, C.T.; Weisel, G.J.; Weiss, B.L.; Zimmerman, D.T. Systematic study of microwave absorption, heating, and microstructure evolution of porous copper powder metal compacts. J. Appl. Phys. 2007. [CrossRef]

6. Ahn, J.H.; Lee, J.N.; Kim, Y.C.; Ahn, B.T. Microwave-induced low-temperature crystallization of amorphous Si thin films. Curr. Appl. Phys. 2002, 2, 135-139. [CrossRef]

7. Birnboim, A.; Calame, J.P.; Carmel, Y. Microfocusing and polarization effects in spherical neck ceramic microstructures during microwave processing. J. Appl. Phys. 1999, 85, 478-482. [CrossRef]

8. Chen, G.; Liss, K.D.; Cao, P. An in situ Study of NiTi Powder Sintering Using Neutron Diffraction. Metals 2015, 5, 530-546. [CrossRef]

9. Grupp, R.; Nöthe, M.; Kieback, B.; Banhart, J. Cooperative material transport during the early stage of sintering. Nat. Commun. 2011. [CrossRef] [PubMed]

10. Bale, H.A.; Haboub, A.; MacDowell, A.A.; Nasiatka, J.R.; Parkinson, D.Y.; Cox, B.N.; Marshall, D.B.; Ritchie, R.O. Real-time quantitative imaging of failure events in materials under load at temperatures above $1600{ }^{\circ} \mathrm{C}$. Nat. Mater. 2013, 12, 40-46. [CrossRef] [PubMed]

11. Xu, F.; Li, Y.; Hu, X.; Niu, Y.; Zhao, J.; Zhang, Z. In situ investigation of metal's microwave sintering. Mater. Lett. 2012, 67, 162-164.

12. Li, Y.C.; Xu, F.; Hu, X.F.; Kang, D.; Xiao, T.Q.; Wu, X.P. In situ investigation on the mixed-interaction mechanisms in the metal-ceramic system's microwave sintering. Acta Mater. 2014, 66, 293-301.

13. Kuczynski, G.C. Self-diffusion in sintering of metallic particles. AIME Trans. 1949, 185, 169-178.

14. Gonzalez, R.C.; Woods, R.E.; Eddins, S.L. Digital Image Processing Using Matlab; Publishing House of Electronics Industry: Beijing, China, 2005; pp. 315-317.

15. Cheng, J.; Roy, R.; Agrawal, D. Experimental proof of major role of magnetic field losses in microwave heating of metal and metallic composites. J. Mater. Sci. Lett. 2001, 20, 1561-1563. [CrossRef]

16. Huang, Y. Electromagnetic Field and Microwave Technology, 1st ed.; Posts \& Telecom Press: Beijing, China, 2007; pp. 77-101.

17. Feng, C. Electromagnetic Field, 2nd ed.; Higher Education Press: Beijing, China, 1983; pp. 322-362.

18. Tracy, M.; Groza, J.R.; Yamazaki, K.; Sudarshan, T.S. Preliminary studies on the densification of fine tungsten powders by plasma activated sintering (PAS) process. In Proceedings of the 2nd International Conference on Tungsten and Refractory Metals, McLean, VA, USA, 17-19 October 1994; pp. 291-297.

(C) 2016 by the authors; licensee MDPI, Basel, Switzerland. This article is an open access article distributed under the terms and conditions of the Creative Commons by Attribution (CC-BY) license (http:/ / creativecommons.org/licenses/by/4.0/). 